\title{
LA ADMINISTRACIÓN CIENTÍFICA DE LA EDUCACIÓN Y LA CONDICION CONTINGENTE DEL SER HUMANO
}

\author{
MANUel Losada-SierRA ${ }^{1}$
}

RESUMEN: La administración científica de la educación ha llevado a que las instituciones educativas muestren en los últimos años un marcado interés por los rankings, la rendición de cuentas y por una educación de calidad basada en la excelencia individual. Frente a esta dinámica, el presente trabajo muestra que las exigencias de una sociedad que ha venido de experiencias conflictivas o que quiere formar ciudadanos solidarios, requieren un cambio de perspectiva desde la autonomía individual hacia la construcción de escenarios de cooperación y respeto por el otro. Mediante un análisis teórico, este artículo muestra la insuficiencia del modelo taylorista de educación y propone una educación para la incertidumbre como apertura hacia la cooperación y la solidaridad.

Palabras-clave: Razón. Víctimas. Incertidumbre. Cooperación.

\section{THE CONTINGENT CONDITION OF THE HUMAN BEING AND THE SCIENTIFIC ADMINISTRATION OF EDUCATION}

\begin{abstract}
The scientific administration of education has led educational institutions to show, in recent years, a strong interest in rankings, accountability, and quality education based on individual excellence. Faced with this dynamic, the present work shows that, in a society, the demands which have come from conflicting experiences or that want to form sympathetic citizens require a change of perspective from individual autonomy towards the construction of cooperation and respect scenarios for the other. Through a theoretical analysis, this article shows the inadequacy of the Taylorist model of education and proposes an education for uncertainty as an opening towards cooperation and solidarity.
\end{abstract}

Keywords: Reason. Victims. Uncertainty. Cooperation. 


\title{
A CONDIÇÃO CONTINGENTE DO SER HUMANO E A ADMINISTRAÇÃO CIENTÍFICA DA EDUCAÇÃO
}

\begin{abstract}
RESUMO: A administração científica da educação levou as instituições educacionais a mostrar, nos últimos, anos um interesse acentuado em rankings, prestação de contas e educação de qualidade com base na excelência individual. Diante dessa dinâmica, o presente trabalho mostra que as demandas de uma sociedade advindas de experiências conflitantes ou que querem formar cidadãos solidários requerem uma mudança de perspectiva da autonomia individual para a construção de cenários de cooperação e respeito ao outro. Por meio de uma análise teórica, este artigo mostra a inadequação do modelo de educação taylorista e propõe uma educação para a incerteza como abertura à cooperação e à solidariedade.
\end{abstract}

Palavras-chave: Razão. Vítimas. Incerteza. Cooperação.

\section{Introducción}

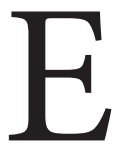

ste artículo defiende la necesidad de educar para la compasión y la cooperación frente al marcado énfasis que las instituciones de educación hacen en el perfeccionamiento individual, la formación por competencias y la excelencia académica. En efecto, la cultura de los rankings que se está imponiendo en el mundo académico, ha hecho que las instituciones se preocupen por competir entre ellas a fin de lograr figuración y posicionamiento social. En este sentido, se ha creado una cultura de control y rendición de cuentas propias de los sistemas de inspección y vigilancia, la cual termina asumiendo modelos productivos de corte taylorista que buscan estructurar científicamente la administración educativa con el fin de producir resultados que sean reconocidos y auditados por el mismo sistema económico que los anima. Cope y l'Anson anotan que "dentro de este proyecto, la educación ha sido discursivamente restructurada según la lógica del mercado, en donde las políticas educativas se han visto crecientemente colonizadas por las iniciativas políticas de la economía" (2003, p. 219), en donde la educación ha sido transformada en medio para la adquisición de conocimiento instrumental y de competencias propias del mercado.

Una consecuencia de este modelo de administración es el marcado interés en diseñar currículos basados en competencias en los cuales está claramente definido "aquello que los estudiantes deberían hacer, en qué condiciones y con cual estándar” (NODDINGS, 1992, p. 4). El objetivo final de estos diseños es la eficiencia en la producción mediante el desarrollo de objetivos y métodos que les permitan a los estudiantes alcanzar ciertos estándares bajo el control de sus profesores, quienes a su vez son controlados por el sistema administrativo escolar. El interés por la eficiencia administrativa y por los resultados finales, lleva al mismo sistema educativo a privilegiar el cultivo de la inteligencia, el perfeccionamiento individual y a favorecer aquellos estudiantes que se muestran más brillantes o prometedores en términos de resultados académicos estandarizados (CROCCO; COSTIGAN, 2006; ROSENBUSCH, 2005; PEDULLA et al., 2003). Este interés se refleja en la famosa frase del presidente de la Universidad de Chicago, Robert Maynard Hutchins, quien expresó: "la mejor educación para los mejores es la mejor educación para todos" (MEYER, 2012). La preocupación por la eficiencia se muestra en la cultura de la "excelencia académica", la cual se mide en la actualidad a través de los rankings y las clasificaciones.

Esta manera de ver la educación termina olvidando la condición contingente del ser humano (SMITH, 2006, p. 25; MORIN, 2001; WAIN, 2006), y, por tanto, ignora la necesidad de educar para las incertidumbres como camino para una educación que tenga en cuenta la compasión y la cooperación como 
caminos para enfrentar la vida en común. Creemos que la visión científica de la administración aplicada a la educación es insuficiente y que el proceso educativo debe ayudar también a formar para superar los conflictos mediante una real preocupación por los otros particularmente con el sufrimiento de aquellos que han padecido violencia.

La crítica al taylorismo no significa que descartemos la necesidad de procedimientos administrativos en la gestión escolar. Estos procedimientos son necesarios y útiles en el propósito de alcanzar el bienestar sicológico, mental y emocional de los estudiantes. Nuestra apuesta es por generar un espacio de reflexión crítica cuando en el sistema escolar lo administrativo ocupa la posición central y definitoria de lo que se debe hacer en las aulas de clase.

Muchos estudios han relacionado la búsqueda de la excelencia y el control administrativo a nivel escolar con la salud sicológica de los estudiantes. En efecto, varios estudios han encontrado cómo la necesidad de mostrar resultados en exámenes es una fuente significado de presión y estrés para los estudiantes. Algunos de estos estudios se enfocan en mostrar el problema desde sus implicaciones individuales dependientes del género y de la autoestima de los estudiantes (ASELTON, 2012; PUTWAIN, 2009; AHERNE 2001) sugiriendo que algunos tipos de estudiantes son especialmente inclinados a la ansiedad frente a las exigencias de resultados. Otros estudios muestran el impacto que tienen en el bienestar emocional de los estudiantes la aplicación de exámenes y la necesidad de responder a las expectativas de padres y profesores (ASSANA et al., 2017; BANKS; SMYTH, 2015; BLODGETT et al., 2012). Creemos que la problemática planteada en estos estudios es real y afectan a muchos estudiantes que se ven enfrentados a responder a la exigencia de resultados. Sin embargo, falta todavía mucho por decir en torno a los efectos que el proceso de control administrativo y el énfasis en los resultados tienen en el proceso formativo de los estudiantes en términos de convivencia y cooperación, particularmente en sociedades que se han visto sometidas a ciclos de violencia prolongada como es el caso de Colombia.

Para responder a esta preocupación, el cuerpo del artículo estará dividido en tres partes. En la primera problematizaremos la presencia de la administración científica de corte taylorista en las instituciones de educación y las consecuencias que tienen en el ambiente escolar. En la segunda parte, argumentaremos la necesidad de enfatizar lo contingente y limitado de la naturaleza humana en los procesos educativos frente al énfasis en el perfeccionamiento personal que ha sido característico de los procesos tayloristas de administración. Finalmente, concluiremos cómo la centralidad que ha adquirido el discurso de la excelencia y el exagerado poder que los controles administrativos han adquirido en la educación alejan a los estudiantes de las experiencias humanas inmediatas como la falibilidad y la necesidad de abrir espacios de cooperación con los otros.

La perspectiva del trabajo es filosófica y se ubica dentro de la reflexión teórica de la filosofía de la educación. Esto significa que no buscamos plantear propuestas pedagógicas prácticas o cambios curriculares particulares en el planeamiento educativo. Queremos fundamentar una reflexión sobre el quehacer educativo con el fin de motivar cambios en la organización de nuestro trabajo educativo.

\section{La Administración Científica de la Educación: Insuficiencia de la Racionalidad Técnica}

\section{Centralidad de la Administración Educativa}

La administración científica de la educación propia del taylorismo está anclada una racionalidad técnica que privilegia aspectos procedimentales sobre los transformativos en el proceso educativo. En otras 
palabras, se privilegian los resultados de un proceso de control sobre el aprendizaje más que el acompañamiento del educando en su proceso de maduración mental, afectiva, emocional y social.

En efecto, el taylorismo introdujo en la educación el concepto de eficiencia y control como mecanismo para mantener el orden y la responsabilidad por los resultados en las instituciones de educación y, por tanto, es uno de los factores de la existencia de los mecanismos de jerarquización (ranking) de las instituciones de educación en la actualidad (AU, 2011). En este sentido, entendemos que las relaciones capitalistas presentes en la educación son más amplias y complejas. Sin embargo, nuestro propósito es inicialmente genealógico, y, por tanto, buscamos explorar los orígenes del fenómeno de control, jerarquización y búsqueda de eficiencia en las instituciones de educación. Trataremos además de mostrar cómo este interés es insuficiente y perjudicial cuando se trata de una educación que responda a los retos de construir cooperación, solidaridad y convivencia pacífica en las sociedades contemporáneas permeadas por la competencia y la violencia.

Para empezar, digamos que la administración científica del trabajo propuesta por Frederick Winslow Taylor se caracteriza en términos generales por concentrar el trabajo en tareas cuantificables, en medir sus resultados, en ejercer un control directivo sobre los trabajadores, y finalmente en privilegiar las buenas prácticas sistemáticamente planificadas. En efecto, una de las más importantes características de la administración científica es la idea de que el trabajo de cada empleado debe ser completamente planeado por anticipado. Esta planeación debe especificar no solamente lo que debe ser hecho sino también cómo y en qué tiempo debe ser realizado. Cada vez que el trabajador tiene éxito en realizar correctamente el trabajo y dentro del tiempo establecido, es recompensado por su esfuerzo.

Los criterios tayloristas de monitorear, evaluar y competir con el propósito de obtener mayor eficiencia, muy pronto permearon los sistemas educativos de la mano de educadores como Frank Spalding, John Franklin Bobbit y Edward Lee Thorndike. Estos pensadores abogaban por la implementación de la administración científica de Taylor en las instituciones de educación, con el mismo propósito, es decir, lograr eficiencia y resultados. No faltaron voces también provenientes de economistas, así como de periódicos y revistas (CALLAHAN, 1962; CUBAN, 2004) que pedían a los administradores educativos que aprovecharan las lecciones de las empresas para elaborar mediciones que permitieran evaluar los resultados y "productos" de la academia. En este sentido, Trujillo afirma: "monitorear, examinar, y competir, pronto permeó la educación pública, y la práctica de contratar "expertos eficientes" para recoger información acerca del desempeño de las escuelas, y hacer recomendaciones para maximizar la productividad se volvió un lugar común” (TRUJILLO, 2014, p. 4; ver STOLLER, 2015).

Desde el punto de vista educativo, estos resultados están asegurados por una autoridad administrativa centralizada que monitorea el cumplimiento de objetivos, previamente diseñados por los administradores, para todas las tareas desarrolladas dentro de las instituciones de educación (IREH, 2016, p. 11). Como Bobbit argumenta,

son los objetivos [...] los que determinan los métodos específicos usados por los profesores, lo mismo que el material didáctico, las herramientas y las oportunidades de aprendizaje que se usen. Estos objetivos determinan igualmente la supervisión, la naturaleza de la organización supervisora, la cantidad de presupuesto, y todas las funciones involucradas en la obtención de los resultados. Y finalmente son los objetivos específicos los que proporcionan los estándares que se usarán en la evaluación de los resultados (BOBBITT, 1912, p. 142).

Desde esta perspectiva, los administradores deben reunir toda la información acerca del proceso educativo con el fin de desarrollar los mejores métodos que deberán ser usados por los docentes con el fin de 
que los estudiantes alcancen los estándares previamente establecidos. Estos métodos deben contar además con criterios de medición que permitan evaluar los productos de la labor escolar y al mismo tiempo emplear la lógica económica para guiar el emprendimiento educativo (TRUJILLO, 2014). De lo dicho se deduce que la aplicación del método científico de administración en el sistema educativo ha tenido como consecuencia el desarrollo y fortalecimiento del control institucional y de la autoridad administrativa. En efecto, si las instituciones educativas quieren promover el desempeño y los resultados de los estudiantes, se tienen que adoptar mecanismos de control por parte de quienes administran con el fin de asegurar que se alcancen dichos resultados. Como parte de este mismo mecanismo administrativista, se ha hecho énfasis en los resultados medidos a partir de pruebas estandarizadas, los cuales se convierten a la vez en medida de la calidad en la educación. Al final, los resultados de estas pruebas van a permitir el posicionamiento institucional en los rankings.

Como consecuencia de este interés por los resultados, se ha afianzado una "cultura de la auditoría" (APPLE, 2007; POWER, 2003) en los centros educativos. Los auditores de calidad se enfocan en verificar el cumplimiento de los estándares que aseguren la consecución de resultados, y por tanto su objetivo es juzgar la eficiencia y efectividad tanto de los docentes como de las instituciones de educación. La administración científica requiere una constante producción de evidencia de que los docentes y administradores educativos están haciendo las cosas de forma "eficiente" y en la forma "correcta".

Apple llama la atención sobre las consecuencias que la cultura de la auditoria genera sobre aquellos que son auditados, es decir, sobre la identidad misma de los educadores. En efecto, Apple al invocar la frase de Margaret Thatcher, de que "el objetivo no es solamente cambiar la economía, sino también el alma" (2007, p. 6) enfatiza que la cultura de la auditoría no solamente produce nuevas prácticas de medición, sino que los auditados se vuelven dependientes de estas mediciones y necesitados de conocer los resultados que la auditoría muestra sobre su propio desempeño. Existe el riesgo además de que educadores y administradores educativos se alineen con los valores y creencias de las lógicas economicistas con tal de mantenerse laboralmente en una sociedad orientada por el mercantilismo y la competencia económica. Una de las características del interés por resultados es, por tanto, la consolidación del "culto de la eficiencia" y de la excelencia en la educación (WELCH, 1998; TRUJILLO, 2014).

Este interés por la eficiencia es usado con frecuencia como argumento legitimador del interés por fortalecer la educación privada a expensas de la pública. Un ejemplo de esta postura es la tesis con que grupos cercanos al partido político colombiano Centro Democrático justifican la propuesta de que los niños estudien en colegios privados pagados por el estado, en vez de asistir a colegios públicos. Lo explican basados en que "los niños colombianos tienen derecho a recibir educación de calidad, y los colegios privados son una garantía de esos derechos" (El Tiempo, 2019). En otras palabras, la educación pública es poco eficiente frente al costo que supone para el Estado, además de ser un escenario de "adoctrinamiento". Por eso, "educación sin adoctrinamiento, pagada por el Estado a los de menos ingresos, con competencia” (JEREZ, 2019). La asunción de que el sector privado es más eficiente que el púbico y más prístino en términos ideológicos se legitima por la conformidad que mantiene con los cánones de la eficiencia económica. Esta eficiencia es promovida a expensas de la consideración fundamental de la educación como un servicio que el estado debe prestar a toda la comunidad, y como un derecho de los ciudadanos.

El énfasis que la administración científica pone en la eficiencia y en los resultados, está relacionado con la prevalencia de una mentalidad tecnocrática que se ha afianzado en la modernidad. Esta tendencia, propia de las sociedades capitalistas, se distingue por una gran preocupación por lo técnico, es decir, por lo administrativo y lo industrial; mientras las consideraciones éticas quedan relegas y supeditadas a lo productivo. Esta característica fue vista y analizada especialmente por la teoría crítica de la Escuela de Frankfurt. 
Pensadores de la teoría crítica como Jürgen Habermas y Herbert Marcuse son críticos de la forma como la conciencia tecnocrática ha colonizado nociones como bien común, libertad y emancipación. Lo técnico define el sistema de valores y la misma ética en nombre de la libertad. En efecto, el criterio de la racionalidad técnica es "la manipulación y el control, el cálculo y la utilidad" (LOSADA SIERRA, 2017, p. 27). En este proceso, los fines y los medios de la eficiencia económica permean las instituciones sociales y las prácticas a expensas de la ética y del bien común (WELCH, 2016). Desde la perspectiva académica, Ball (1990) muestra cómo en el contexto educativo la eficiencia económica es simplemente asumida sin ser problematizada como parte precisamente del incremento en el uso de tecnologías de control.

Esta preocupación de la Escuela de Frankfurt es compartida por el filósofo Jean-François Lyotard quien en su concepción de la posmodernidad encuentra que lo performativo está presente en distintas estructuras de la sociedad, entre ellas la educativa. En la sociedad moderna, el conocimiento se transforma en un commodity y se convierte en una fuerza productiva. Por esta razón, el trabajo académico está sujeto a un trabajo de performatividad en el que se busca optimizar su desempeño desde lo económico y administrativo (LYOTARD, 1984, p. xxiv; ver HOYOS VÁSQUEZ, 2007). Para Lyotard las preguntas de la eficiencia administrativa se vuelven comunes en el campo educativo. Por ejemplo, ¿es eficiente? o ¿es vendible? se vuelven más importantes que ¿es verdad? (LYOTARD, 1984, p. 51).

Vale la pena analizar entonces algunas consecuencias que se derivan de la administración científica de la educación y de la prevalencia de criterios administrativos y económicos en el manejo de las instituciones de educación.

\section{Consecuencias de la Administración Científica de la Educación}

Son varias las consecuencias que se pueden deducir del uso de este tipo de paradigma en la educación. En primer lugar, si consideramos que los test estandarizados son una tecnología, una herramienta para medir y comparar estudiantes en situaciones específicas, ellos ponen muy poca o ninguna atención a las diferencias individuales. En efecto, los test son considerados objetivos porque supuestamente miden a todos los individuos en igualdad de condiciones, sin embargo, estas mediciones no tienen en cuenta circunstancias diferenciadoras y de contexto. Por tanto, cuando los estudiantes sin medidos por test estandarizados y comparados con otros estudiantes, ellos son necesariamente descontextualizados con el fin de poder hacer posible dicha comparación (AU, 2011). Por esta razón, John Dewey buscaba disuadir a los responsables de los procesos educativos de usar principios tayloristas. Para él el uso de pruebas desde la perspectiva de la administración científica es un mecanismo de clasificación y estandarización, mientras que las pruebas deberían, en su perspectiva, ser usados para conocer mucho mejor a los estudiantes (CALLAHAN 1962).

Además, la administración científica de la educación genera una pérdida de autonomía de los docentes y de los estudiantes en su proceso educativo. En efecto, el monitoreo que realiza la administración de lo que sucede en el aula de clase y de sus resultados, termina por obligar a los docentes a adecuar lo que enseñan a los que se espera que los estudiantes demuestren en los exámenes estandarizados (AU, 2011). La creatividad, la generación de ideas y las capacidades de estudiantes y docentes terminan marchitándose bajo la búsqueda de efectividad en el trabajo docente (BRIDWELL-MITCHELL, 2015).

Aparte de las consecuencias arriba mencionadas, creemos que el énfasis en los resultados como fruto de este paradigma administrativista ha privilegiado una visión individualista del ser humano. En efecto, la estructuración del sistema educativo alrededor de resultados medibles en el proceso de aprendizaje ha generado competencia y búsqueda de perfección individual (TRUJILLO, 2014; LIPMAN, 2011). Al fin de 
cuentas lo que hace el sistema es monitorear y evaluar el desempeño individual de los estudiantes frente a los resultados esperados. Funcionalmente, estas pruebas determinan quién pasa y quién pierde en el proceso educativo con el fin, a su vez, de determinar quién merece una recompensa, normalmente una calificación cuantitativa, y también, quién amerita una sanción. Como afirma Welch "los seres humanos son vistos con mayor o menor valor dependiendo de su nivel de resultados y de sus talentos" (1998, p. 158).

Por último, creemos que el énfasis en los resultados fruto de la concepción administrativista de la educación ha creado una concepción abstracta de la educación en la que se desconoce la condición más característica de la vida humana, es decir, la condición de contingencia y vulnerabilidad de la vida humana (HOYOS VÁSQUEZ, 2007). El énfasis en la perfección crea individuos alejados de los intereses comunes y de las exigencias de cooperación social, más cercanos a los requerimientos de una "sociedad transparente" (Vattimo) que a las exigencias de una sociedad en crisis necesitada de la participación cooperativa de todos sus miembros.

Por esa razón, creemos que una educación que haga énfasis en sistemas de control administrativo y en resultados no está respondiendo al reto de formar ciudadanos cooperativos para una sociedad individualista marcada por la violencia, el afán de éxito y la preocupación exclusiva por el crecimiento económico. Educar para la cooperación requerirá recordar la condición contingente y finita de la naturaleza humana que abra las puertas al reconocimiento del otro, particularmente del que sufre y requiere de nuestra compasión y solidaridad.

\section{Educar para la Contingencia y la Cooperación}

Uno de los autores que con mayor énfasis critica esta perspectiva de la educación que enfatiza los logros académicos y el énfasis en la formación racional del estudiante es el educador inglés Richard Smith. Según Smith, la visión administrativista de la educación con sus mediciones y logros refleja una cultura racional, calculadora y utilitarista, que nos hace olvidar la importancia de pensar lo contingente y finito de la naturaleza humana. Esta visión es la respuesta a la exagerada confianza que tenemos en nuestra capacidad intelectual y en nuestra habilidad para hacer que el mundo responda a nuestros deseos.

Como decíamos atrás, esta visión crítica de la racionalidad calculadora y utilitarista fue también preocupación de los miembros de la Escuela de Frankfurt, quienes fueron igualmente críticos de la racionalidad instrumental y dominadora que surgió de la ilustración y que venía gestándose desde el renacimiento como fruto de la centralidad que empezó a tener el ser humano en el pensamiento filosófico. Para filósofos como Adorno y Horkheimer (1998) los criterios de la racionalidad instrumental son los de la manipulación, el control, el cálculo y la utilidad, y por eso para ella resulta sospechoso todo aquello que no sea computable y útil. En este sentido, la racionalidad técnico-científica termina en contra del mismo individuo en cuanto reduce su misma interioridad a la condición de ser objeto de dominio.

En relación con esta reducción de la interioridad del sujeto, Horkheimer llama la atención sobre otra consecuencia del predominio de esta racionalidad (ADORNO; HORKHEIMER, 1998). Se refiere a la pérdida del sentido de verdad en términos éticos. Para él, ideas como justicia, libertad y tolerancia han perdido su relación con la razón y por tanto no existe una racionalidad que las aprecie y las relacione con la realidad objetiva. La razón se vuelve entonces instrumental en la medida en que es aceptada en su forma operacional que se enfoca en la dominación del hombre sobre la naturaleza. Además, el predominio del empirismo y cientificismo consideran la verdad como un problema de procedimiento, y por tanto verificable y demostrable empíricamente. 
La instrumentalización de la razón, la imposibilidad de fundamentar verdades universalmente válidas y la mercantilización de los valores humanos son inquietudes compartidas también por el teólogo Johann Baptist Metz. Para el teólogo alemán, el privilegio que la modernidad ha concedido a la racionalidad orientada por lo técnico científico ha hecho que con facilidad se pierda la memoria de la violencia y del dolor de los más débiles. Al recordar que el propósito del iluminismo era hacer que el sujeto madurara mediante el pleno uso de sus facultades intelectuales, Metz considera que este sujeto terminó dominado por la razón técnico-científica. Esta razón está marcada por el conocimiento dominador de la naturaleza y, por tanto, el sujeto se entiende a sí mismo ejerciendo control sobre esa misma naturaleza. Así se olvida fácilmente fenómenos catastróficos como el que él mismo vivió durante la segunda guerra mundial. Por eso se pregunta con urgencia: “¿cómo se puede creer en el ser humano o incluso [...] en la humanidad, cuando uno tuvo que ser testigo en Auschwitz de todo lo que el 'ser humano' es capaz? [...] no solo el individuo humano sino las ideas de ser humano y de humanidad son profundamente vulnerables" (METZ, 1980, p. 20). Queda en entredicho entonces el propósito del iluminismo de volver al sujeto más fuerte por su capacidad de pensar con autonomía.

Esta crisis del sujeto se hace evidente en lo que Metz denomina "pequeña moralidad", la cual es reacia a aceptar compromisos y responsabilidades frente a los otros seres humanos, particularmente los más desfavorecidos. Esta pequeña moralidad está presente en la privatización de la vida dentro del proceso de mercantilización de las relaciones humanas y en la ideología del progreso como una visión positivista y tecnocrática de la historia humana.

Culturalmente, el sujeto amenazado de esta pequeña moralidad emerge en una sociedad en la cual los principios de la economía han colonizado la vida humana, y por tanto los principios del intercambio prevalecen en las relaciones humanas. Estos principios dejan a la elección privada e individual todo lo que no se ajusta a la razón calculadora. Desde esta perspectiva no hay lugar para los significados públicos y universales de la memoria, la responsabilidad y la solidaridad. Al contrario, una cultura de apatía y falta de compromiso surge en medio de una sociedad orientada hacia el progreso.

Así, nos hemos convertido gradualmente en simples espectadores de las dificultades y tragedias ajenas. Fácilmente podemos permanecer sin ser afectados por las grandes desigualdades que nos separan a algunos de nosotros de aquellos que luchan desesperadamente para sobrevivir. Esta característica de nuestra realidad social perpetúa la conectividad unilateral, paternalista y no recíproca con los demás. Pocas oportunidades tenemos de auto reflexión sobre nuestras conexiones con el sufrimiento de otros o sobre nuestra complicidad en sus realidades injustas.

El filósofo Emmanuel Lévinas señala cómo la violencia que generaron las guerras del siglo XX mostró la insuficiencia de la "inteligencia moderna" para prevenir la catástrofe, e incluso estuvo comprometida con la guerra y la barbarie. Para él, el hecho de que el humanismo filosófico se haya centrado en la libertad y capacidad autónoma del sujeto ha terminado en egoísmo y autosuficiencia en las relaciones humanas. Un punto ciego que mostró en la aventura hitleriana su más dramática consecuencia. Tal vez por eso Theodor Adorno insistió en que el propósito de la educación consistía en que Auschwitz no se repitiera y que floreciera el respeto por el otro.

Este "fiasco humano", según Lévinas, muestra una inherente aporía: el sujeto humano se siente dueño de sus acciones y decisiones, defiende la libertad y rechaza cualquier intento exterior de alienación. Todo en la vida del sujeto debe ser asumido y reflejado por la conciencia racional, de lo contrario, el sujeto podría perder su propia identidad como un ser libre y racional. Sin embargo, la realidad humana es una limitación constante, un riesgo permanente de muerte, violencia y guerra. De hecho, un sujeto que se siente dueño de sus actos y, por lo tanto, identificado consigo mismo, choca con la realidad de la limitación que significa ser humano. 
El escritor colombiano Abad Faciolince (2006) mientras narra la terrible tragedia que significó la muerte de su padre y la violencia que vivió Medellín, su ciudad natal, se pregunta si la violencia es amorfa, indiscriminada y loca. Razones no le faltan al ver tanto terror desmesurado e indiscriminado en contra de los seres más nobles e indefensos. La respuesta que da es pavorosa: “Todo lo contrario. El actual recurso al asesinato es metódico, organizado, racional” (ABAD FACIOLINE, 2006, p. 280). Tal vez, porque la razón está vinculada al intelecto y este es individual y este nos hace "estrategas, productivos y poderosos" (RODRÍGUEZ, 2005, p. 168). En efecto, el intelecto es sinónimo de grandeza y brillo, mientras la torpeza es signo de debilidad y desventaja. De ahí que "la inteligencia es como un acto de fuerza que nos hace competitivos y nos libra de la debilidad” (PARRA RODRÍGUEZ, 2005, p. 169). Tal vez por eso, Nussbaum (2006) sugiere que el compromiso con la dignidad humana tiene que enfocarse en áreas de vulnerabilidad y no solamente en nuestra capacidad por una autonomía individual.

Basados en las anteriores consideraciones, deberíamos por lo menos sospechar del marcado énfasis que la educación pone en la formación para la excelencia basados en la competencia y la búsqueda de resultados. Creemos que el reto de la educación debería estar más enfocado en enseñar la contingencia y vulnerabilidad de la condición humana y menos en el perfeccionamiento individual.

El reconocimiento de la falibilidad humana abre las puertas a la cooperación y la solidaridad. El sistema escolar está llamado "no tanto a aprender certidumbres sino a educarnos para la tolerancia y la cooperación” (LOSADA SIERRA, 2017, p. 29).

La misma ciencia desde distintas perspectivas ha reconocido las limitaciones y la vulnerabilidad de la condición humana. Por ejemplo, a pesar de que la imagen que nos ha llegado de Darwin es la del defensor de la certeza en cuanto que la selección natural asegura lo mejor porque solamente lo mejor subsiste, él mismo reconoce que las imperfecciones son la marca del progreso histórico y que la naturaleza más que encarnar leyes inexorables es "torpe" y "propensa a los accidentes". De igual forma, el principio de Heisenberg nos recuerda que no podemos tener seguridad y certeza absoluta sobre los eventos físicos.

Esto significa que en nuestro conocimiento debemos tener siempre tolerancia frente a la incertidumbre. El conocimiento debe ser tratado con humildad porque es imperfecto, como imperfecta es la condición humana. Estamos inmersos en la incertidumbre y esto nos obliga a pensar que el intercambio de información y de conocimiento entre los seres humanos solo puede darse en un ámbito de tolerancia. Esta constatación nos lleva a negar el conocimiento absoluto, sea este científico, político o religioso.

Desde lo ético, esto también significa que no podemos establecer juicios sobre los demás basados en algún tipo de conocimiento cierto. Los fundamentalismos y dogmatismos son usualmente el resultado de certezas, las cuales llevan a la arrogancia y a la violencia. Las relaciones sociales requieren un principio de tolerancia en el que no tienen cabida posiciones absolutistas e irrenunciables. Como afirma Harari, "las personas que temen perder su verdad tienden a mostrarse más violentos que las personas acostumbradas a considerar el mundo desde distintos puntos de vista" (HARARI, 2018, p. 231-232).

Se requiere, por tanto, un cambio en la educación desde un paradigma de competitividad hacia el ejercicio de la cooperación y la práctica de la solidaridad. Desde una educación enmarcada en el paradigma de la precisión, la eficiencia y la positivización hacia una educación que parta del reconocimiento de lo típicamente humano como falible. En este sentido, Adorno manifestaba como la competencia que se genera en los procesos educativos es, en el fondo, un principio opuesto a una educación humana: "creo además también que una enseñanza que discurre en formas humanas no puede tender en absoluto a reforzar el instinto de competencia”. Y agregaba cómo “convendría un modelo de educación en el escepticismo frente a la 'sana' voluntad de éxito" (ADORNO, 1998, p. 110).

En este sentido, es significativo el giro que el sistema educativo de Singapur ha empezado a dar 
desde el año 2019. Como sabemos, Singapur se ha caracterizado por un fuerte énfasis en el éxito de los estudiantes al presentar los exámenes estandarizados, particularmente los que resultan de las pruebas PISA. Ahora, el gobierno de Singapur considera que no se puede seguir clasificando a los estudiantes según los resultados porque como afirma su ministro de educación Ong Ye Kung, "aprender no es una competición" (WOOD, 2018). La competición ha hecho que los estudiantes estén más atentos a ganar y menos interesados en aprender. En el mismo sentido y por las mismas razones, el sistema finlandés no usa pruebas estandarizadas sino pruebas individuales que permitan hacer un seguimiento al aprendizaje basado en las capacidades e intereses de cada estudiante. Pero lo que es aún más importante, el sistema busca enfatizar la cooperación antes que la competición (DICKINSON, 2019).

\section{Consideraciones Finales}

Nos planteamos como problemática la necesidad de revaluar el marcado énfasis que las instituciones de educación y el sistema de educación en general han mostrado en relación con la administración científica de la educación. El problema que vimos es la falta de espacio para educar en la falibilidad y las limitaciones de la condición humana. Creemos que reconocer y tener en cuenta esta condición de imperfección es el camino para educar en la solidaridad y la cooperación frente al individualismo que se genera cuando se enfatiza la perfección individual y la obtención de resultados. La fundamentación que hemos hecho para guiar la reflexión ha sido teórica y la hemos enmarcado dentro de la filosofía de la educación.

Este planteamiento nos ha llevado a revisar las preocupaciones que han tenido algunos investigadores sobre el control que la prevalencia de lo administrativo ha tenido en los últimos años en relación con los resultados que se exigen a los estudiantes en los exámenes. De igual manera, hemos revisado de forma crítica el valor que las instituciones han concedido a los rankings y clasificaciones. Estos dos fenómenos están estrechamente relacionados en cuanto que normalmente los rankings dependen de los resultados que los estudiantes muestren durante y al final de los procesos educativos.

Creemos que la aversión al error y al aprendizaje que de él se puede obtener, ha llevado al sistema educativo a desarrollar un carácter manipulador, una manía organizativa y un sobredimensionamiento de la excelencia como objetivo de lo educativo que deriva en una incapacidad para tener experiencias humanas inmediatas. Creemos que esto degenera en un culto de la eficiencia y en la voluntad de hacer las cosas que así lo demuestren.

\section{Referencias}

ABAD FACIOLINCE, H. El olvido que seremos. Bogotá: Planeta, 2006.

ADORNO, T. Educación para la emancipación. Conferencias y conversaciones con Hellmut Becker (1959-1969). Madrid: Morata, 1998.

ADORNO, T.; HORKHEIMER, M. Dialéctica de la ilustración. Madrid: Trotta, 1998.

AHERNE, D. Understanding student stress: A qualitative approach. Irish Journal of Psychology, v. 22, n. 3, p. 176-187, 2001. https://doi.org/10.1080/03033910.2001.10558278 
APPLE. M. W. Education, Markets, and an Audit Culture. International Journal of Educational Policies, v. 1, n. 1, p. 4-19, 2007. https://doi.org/10.1111/j.0011-1562.2005.00611.x

ASELTON, P. Sources of stress and coping in American college students who have been diagnosed with depression. Journal of Child \& Adolescent Psychiatric Nursing, v. 25, n. 3, p. 119-123, 2012. https://doi. org/10.1111/j.1744-6171.2012.00341.x

ASSANA, S.; LAOHASIRIWONG, W.; RANGSEEKAJEE, P. Quality of life, mental health and educational stress of high school students in the northeast of Thailand. Journal of Clinical \& Diagnostic Research, v. 11, n. 8, 2017. https://doi.org/10.7860/JCDR/2017/29209.10429

AU, W. Teaching under the new Taylorism: High-stakes testing and the standardization of the 21 st century curriculum. Journal of Curriculum Studies, v. 43, n. 1, p. 25-45, 2011. https://doi.org/10.1080/0022027 2.2010 .521261

BALL, S. Management as moral technology. A Luddite analysis. In: BALL S. (ed.). Foucault and education, disciplines and knowledge. London: Routledge, 1990, p. 153-166.

BANKS, J.; SMYTH, E. 'Your whole life depends on it': Academic stress and high-stakes testing in Ireland. Journal of Youth Studies, v. 18, n. 5, p. 598-616, 2015. http://doi.org/10.1080/13676261.2014.992317

BLODGETT SALAFIA, E.; LEMER, J. Associations between multiple types of stress and disordered eating among girls and boys in middle school. Journal of Child \& Family Studies, v. 21, n. 1, p. 148-157, 2012. https://doi.org/10.1007/s10826-011-9458-Z

BOBBITT, J. F. The elimination of waste in education. The Elementary School Teacher, v. 12, n. 6, p. 259$271,1912$.

BRIDWELL-MITCHELL, E. N. Theorizing teacher agency and reform: how institutionalized instructional practices change and persist. Sociology of Education, v. 88, n. 2, p. 140-159, 2015. https://doi. org/10.1177/0038040715575559

CALLAHAN, R. Education and the cult of efficiency: A study of the social forces that have shaped the administration of the public schools. Chicago: University of Chicago Press, 1962.

COPE, P.; 1'ANSON, J. Forms of exchange: Education, economics and the neglect of social contingency. British Journal of Educational Studies, v. 51, n. 3, p. 219-232, 2003. Disponible en: http://www.jstor.org/ stable/1555869. Acceso em: 30 jul. 2019.

CROCCO, M. S.; COSTIGAN, A. T. High-stakes teachings: What's at stake for teachers (and students) in the age of accountability. The New Educator, v. 2, n. 1, p. 1-13, 2006. https://doi.org/10.1080/15476880500486061

CUBAN, L. The blackboard and the bottom line: Why schools can't be businesses. Cambridge: Harvard University Press, 2004.

DICKINSON, K. How does Finland's top-ranking education system work? World Economic Forum, 2019. Disponible en: https://www.weforum.org/agenda/2019/02/how-does-finland-s-top-ranking-educationsystem-work. Acceso en: 30 jul. 2019. 
HARARI, Y. N. 21 lecciones para el siglo XXI. Barcelona: Debate, 2018.

HOYOS VÁSQUEZ, G. Comunicación, educación y ciudadanía. In: HOYOS VÁSQUEZ, G. et al. Borradores para una filosofía de la educación. Bogotá: Siglo del Hombre Editores, 2007, p. 13-86.

IREH, M. Scientific management still endures in education. Report Winston-Salem State University, 2016. Disponible en: https://eric.ed.gov/?id=ED566616. Acceso en: 30 jul. 2019

JEREZ, D. Uribe propone fortalecer educación privada por 'adoctrinamiento' en colegios. La FM, Bogotá, 2019. Disponible en: https://www.lafm.com.co/politica/uribe-propone-fortalecer-educacion-privada-poradoctrinamiento-en-colegios. Acceso en: 30 jul. 2019.

LIPMAN, P. The new political economy of urban education: Neoliberalism, race and the right to the city. New York: Routledge, 2011.

LOSADA SIERRA, M. Educar para la compasión y la cooperación. In: NAJJAR, J.; CABRALES, O.; DÍAZ, V. (orgs.). Pesquisas em educação no contexto Colombia- Brasil. Curitiba: Appris, 2017, p. 25-36.

LYOTARD, J. F. The postmodern condition. A report on knowledge. Minneapolis: University of Minnesota Press, 1984.

METZ, J. B. Faith in history and society: Toward a practical fundamental theology. New York: The Seabury Press, 1980.

MEYER. P. The best education for the best is the best education for all. Huffpost, 2012. Disponible en: https:// www.huffpost.com/entry/the-best-education-for-th_b_1865074. Acceso en: 2 ago. 2019.

MORIN, E. Los siete saberes necesarios para la educación del futuro. Bogotá: Cooperativa Editorial Magisterio, 2001.

NODDINGS, N. The challenge to care in schools. New York: Teachers College Press, 1992.

NUSSBAUM, M. Frontiers of justice: Disability, nationality, species membership. Cambridge: Harvard University Press, 2006.

PARRA RODRÍGUEZ, J. El cuidado del intelecto. In MESA, J. A. et al. La educación desde las éticas del cuidado y la compasión. Bogotá: Editorial Pontificia Universidad Javeriana, 2005, p. 165-191.

PEDULLA, J. J. et al. Perceived effects of state-mandated testing programs on teaching and learning: Findings from a national survey of teachers. Boston: National Board on Educational Testing and Public Policy, 2003.

POWER, M. The audit society - Second thoughts. International Journal of Auditing, v. 4, n. 1, p. 111119, 2003. https://doi.org/10.1111/1099-1123.00306

PUTWAIN, D. W. Situated and contextual features of test anxiety in UK adolescent students. School Psychology International, v. 30, n. 1, p. 56-74, 2009. https://doi.org/10.1177/0143034308101850

EL TIEMPO. Que Estado pague educación privada a jóvenes de pocos recursos: Uribe. El Tiempo. Redacción Educación, Bogotá, 7 mar 2019. Disponible en: https://www.eltiempo.com/vida/educacion/uribe-proponeque-estado-pague-educacion-privada-a-jovenes-de-pocos-recursos-urib-335038. Acceso en: 15 jul. 2019. 
ROSENBUSCH, M. H. The No Child Left Behind Act and teaching and learning languages in U.S. Schools. The Modern Language Journal, v. 89, n. 2, p. 250-261, 2005. Disponible em: https://www.jstor.org/stable/3588685. Acceso en: 1 ago. 2019.

SMITH, R. Abstraction and finitude: Education, chance and democracy. Studies in Philosophy and Education, v. 25, n. 1-2, p. 19-35, 2016. https://doi.org/10.1007/s11217-006-6436-9

STOLLER, A. Taylorism and the logic of learning outcomes. Curriculum Studies. v. 47, n. 3, p. 317-333, 2015. https://doi.org/10.1080/00220272.2015.1018328

TRUJILLO, T. The modern cult of efficiency: Intermediary organizations and the new scientific management. Educational Policy, v. 28, n. 2, p. 1-26, 2014. https://doi.org/10.1177/0895904813513148

WAIN, K. Contingency, education, and the need for reassurance. Studies in Philosophy and Education, v. 25, n. 1-2, p. 25- 37, 2006. https://doi.org/10.1007/s11217-006-0001-4

WELCH, A. R. The cult of efficiency in education: Comparative reflections on the reality and the rhetoric. Comparative Education, v. 34, n. 2, p. 157-175, 1998. https://doi.org/10.1080/03050069828252

WELCH, A. R. Audit culture and academic production. Higher Education Policy, v. 29, p. 511-538, 2016. https://doi.org/10.1057/s41307-016-0022-8

WOOD, J. Children in Singapore will no longer be ranked by exam results. Here's why. World Economic Forum, 2018. Disponible en: https://www.weforum.org/agenda/2018/10/singapore-has-abolished-schoolexam-rankings-here-s-why/ Acceso en: 5 jul. 2019.

\section{Sobre el Autor}

Manuel Losada-Sierra es PhD en Filosofía por la Griffith University, en Australia. Profesor titular de la Facultad de Educación y Humanidades de la Universidad Militar Nueva Granada, en Cajicá, Colombia. Investigador principal del Grupo de Investigación Humanitas.

Recibido: 16 Ago 2019

Aceptado: 03 Abr 2020 